\title{
Durchblick mit Folgen
}

\author{
Methoden der Virtuel (VR) und Augmented Reality (AR) erobern in der Medizin \\ neue Einsatzfelder. In den USA wird die Zulassung einer VR-Therapie der Amblyopie, \\ des Krankheitsbildes des trägen Auges, durch die zuständigen Behörden geprüft. \\ Von Michaela Endemann
}

Kognitive Erkrankungen aus dem demenziellen Formenkreis können sich Jahre vor einer Diagnose bemerkbar machen. „Neben den bekannten Störungen der Erinnerungen wird beispielsweise die Abnahme der Riechleistung schon lange als früher Marker für neurokognitive Störungen diskutiert", so Wolfgang Staubmann, Dozent am Institut für Diätologie am FH JOANNEUM in Graz. „Weitere Hinweise können die Veränderung des Gangbildes liefern ebenso wie soziale Isolation."

\section{Je früher, desto besser}

Das interdisziplinäre Team rund um Staubmann arbeitet am Forschungsprojekt „SCOBES-AR“. Das Kürzel steht für „Smart Cognition and Behaviour Screening powered by Augmented Reality", also intelligente Kognition und Verhaltensscreening mittels erweiterter Realität. Ziel des Projektes ist es, möglichst früh Abweichungen der kognitiven oder physischen Leistungsfähigkeit von Menschen ab dem 60. Lebensjahr zu erkennen und gezielt ein präventives Maßnahmenpaket für die betroffenen Personen entwickeln zu können. Staubmann macht aber klar: „Eine Diagnose von Demenz erfolgt nur nach den derzeit gültigen klinischen Kriterien und Standards der Fachgesellschaften."

Die vorhandenen Testsysteme werden bisher nur in den einzelnen unabhängigen Disziplinen untersucht, aber nicht zusammen ausgewertet. Das Team ist dabei, die bestehenden Verfahren zu verknüpfen und technisch zu erweitern. „Die AR- und VR-Technologien werden verwendet, um bereits bestehende Screeningverfahren realitätsnaher durchführen zu können und um Auffälligkeiten objektiv zu speichern", so Staubmann.

\section{Handy, VR-Brille und Software}

Das Screening-Instrument besteht aus einem Tablet-PC, einem Smartphone mit einer Kopfhalterung für AR-Inhalte sowie eine VR-Brille. So werden in „Trail Making Tests“ mithilfe von ARoder VR-Technik Alltagsfertigkeiten wie die Bewältigung einer Einkaufssituation oder das Verhalten im Straßenverkehr überprüft. Beim „DemTect“ werden weitere kognitive Funktionen, z.B. Wahrnehmung, Lernen, Erinnerungsvermögen, Denkvermögen, untersucht. Downloads der Android-App gibt es auf der Projektwebseite von SCOBES-AR. „Die Software wird von uns in einem User Centered Design Prozess entwickelt und an die Hardware angepasst, um eine intuitiv bedienbare Gesamtlösung zu gewährleisten“, erklärt Diätologe Wolfgang Staubmann. In allen Entwicklungsschritten seien sowohl die verschiedenen Gesundheitsberufe als auch die zu screenenden Personen aktiv eingebunden: „Die erhobenen Daten werden an eine bereits bestehende Telehealth-Softwarelösung für die Auswertung übermittelt und visualisiert."

Die Coronakrise verzögerte das Projekt. „Eine größere Studie mit 300 Teilnehmer*innen musste gestoppt werden, kann jetzt aber fortgesetzt werden." Das Projektteam wende sehr strenge Hygienerichtlinien an. „So können weitere Studien und in Folge die Praxistauglichkeit durch freiberufliche Health Professionals wie geplant durchgeführt werden“, so Staubmann. Als wichtigsten Einsatzort von SCOBES-AR sieht der Dozent in Zukunft Primärversorgungszentren, Pflegewohnheime oder Tageszentren. Eine SCOBES-AR-App kann für Android-Smartphones auf der Projektwebseite www.scobes-ar.fh-joanneum.at zum Ausprobieren heruntergeladen werden. //

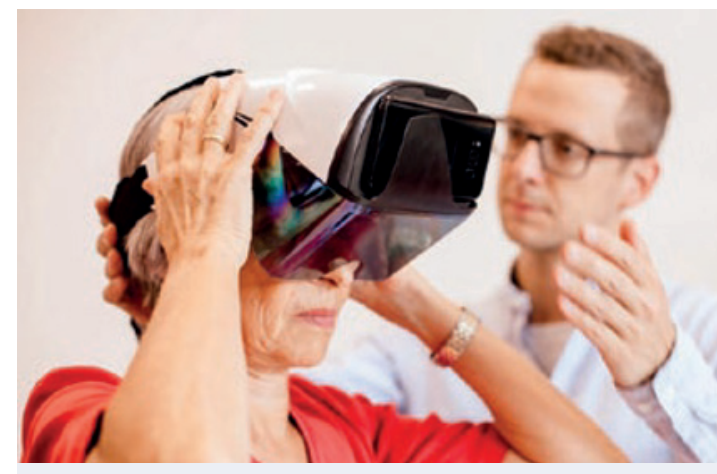

GEEK SPEAK

จ Virtuelle Realität (VR): ist eine computergenerierte Wirklichkeit. Um in diese Welten abzutauchen, benötigt man einen leistungsstarken Rechner und eine Spezialbrille sowie Controller, um in der virtuellen Welt reagieren zu können.

- Augmented Reality (AR): ist eine reale Umgebung mit virtuellen Objekten. Zusätzliche virtuelle Objekte können in der Handykamera über eine Software eingeblendet werden. Der Bezug zur realen Umgebung geht damit nicht verloren wie bei VR. Bekanntestes Beispiel: Pokémon Go. 